\title{
ORIGINAL ARTICLE \\ Muscle perfusion of posterior trunk and lower-limb muscles at rest and during upper-limb exercise in spinal cord-injured and able-bodied individuals
}

\author{
A Zafeiridis ${ }^{1}$, AV Vasiliadis ${ }^{1}$, A Doumas ${ }^{2}, \mathrm{~N} \mathrm{Galanis}^{3}$, T Christoforidis $^{2}$, A Kyparos $^{1}$, MG Nikolaidis ${ }^{1}$, \\ $\mathrm{K}_{\text {Dipla }}{ }^{1}$ and IS Vrabas ${ }^{1}$
}

\begin{abstract}
Study design: Nonrandomized-controlled trial.
Objectives: To assess muscle perfusion at rest and during arm-cranking exercise (ACE) in upper and lower posterior trunk and vastus lateralis $(\mathrm{VL})$ muscles in individuals with spinal cord injury (SCl) and controls (C).

Setting: Exercise Physiology-Biochemistry Laboratory.

Methods: Eight $\mathrm{SCl}$ with thoracic lesion and eight $\mathrm{C}$ received injections of radioactive tracer to trapezius (TRAP), latissimus dorsi (LAT) and VL. Radioactive counts were recorded with a $\gamma$-camera for $10 \mathrm{~min}$ at rest and during ACE $\left(60 \% \mathrm{VO}_{2 \max }\right.$ for $\left.20 \mathrm{~min}\right)$. Timecount curves were generated and the isotope clearance rate, expressed as half-life time ( $\left.T_{1 / 2}, \mathrm{~min}\right)$, was calculated to assess muscle perfusion.

Results: Resting $T_{1 / 2}$ was lower in TRAP and LAT vs VL $(P<0.05)$ in $\mathrm{SCl}$, however, there were no differences among muscles in $\mathrm{C}$. Arm-cranking increased $(P<0.001)$ the isotope clearance in TRAP and LAT in SCl and $\mathrm{C}$, whereas no effect was found on $\mathrm{T}_{1 / 2}$ in VL in both groups. $\mathrm{T}_{1 / 2}$ was longer $(P<0.05)$ in $\mathrm{SCl}$ vs $\mathrm{C}$ in $\mathrm{VL}$ at rest and during $\mathrm{ACE}$, whereas there were no differences between groups in posterior trunk muscles.

Conclusions: Resting muscle perfusion was reduced in the paralyzed limbs of $\mathrm{SCl}$ compared with $\mathrm{C}$, whereas there was no evidence of impaired microcirculation in upper and lower back muscles in SCl. Although ACE did not induce a hyperemic response in VL, it increased hyperemia in upper and lower posterior trunk muscles in $\mathrm{SCl}$, suggesting beneficial effects of this type of activity on muscle microvasculature in this region.
\end{abstract}

Spinal Cord (2012) 50, 822-826; doi:10.1038/sc.2012.36; published online 10 April 2012

Keywords: blood flow, regional; exercise; microcirculation; muscle blood flow; spinal cord injury; arm ergometry test.

\section{INTRODUCTION}

Individuals with a spinal cord injury (SCI) demonstrate structural and functional maladaptations in the microvasculature of their paralyzed limbs, such as reduced capillary diameter and capillarization and increased vascular resistance. ${ }^{1,2}$ Exercise has the potential to reverse these vascular maladaptations through changes in hemodynamic responses during acute exercise bouts. ${ }^{3}$

Arm-cranking exercise (ACE) constitutes a common mode of cardiovascular exercise in SCI. The effects of exercise on vascular remodeling are mainly localized. However, if the hemodynamic stimulus is 'large' and prolonged enough, it may induce systemic effects through increases in anterograde blood flow and shear stress throughout the vasculature. ${ }^{3}$ Indeed, in able-bodied individuals, leg exercise increases blood flow in the arms and the increase in blood flow may induce capillary growth even with negligible increases in metabolism. ${ }^{4}$ Thus, the potential of ACE to enhance blood flow to non-exercising muscles, seems as an attractive intervention to increase the arteriole/capillary diameter, elicit capillarization and diminish the microvascular maladaptations in the paralyzed legs of SCI. The few studies that examined the effects of ACE on leg circulation in $\mathrm{SCI}$ have focused only on changes in limb volume, ${ }^{5}$ cutaneous microcirculation, ${ }^{6}$ and femoral artery blood flow ${ }^{7,8}$ and reported equivocal results. Changes in limb volume and conduit artery blood flow, however, may not entirely reflect changes in muscle perfusion, and the blood flow response between the conduit artery and the microvasculature may vary substantially. Thus, a definite conclusion on the effect of ACE on muscle perfusion in legs of SCI has not been possible.

Upper and lower back muscles are essential in SCI for maintaining trunk stability at rest and performing daily tasks, such as wheelchair propulsion, lateral shifts and body transfers. ${ }^{9}$ During balance perturbations, SCI show lower muscle activation and more frequent involvement of the upper and lower posterior trunk muscles to regain position. ${ }^{10}$ Thus, the improvement or preservation of these muscles' performance is essential in this population. Although the arm and shoulder muscles are primarily involved in ACE, a hyperemic response in low back muscles may induce favorable microvascular adaptations that will improve blood supply and increase performance

${ }^{1}$ Exercise Physiology and Biochemistry Laboratory, Department of Physical Education and Sport Sciences at Serres, Aristotle University of Thessaloniki, Serres, Greece; ${ }^{2}$ Third Department of Nuclear Medicine, General Papageorgiou Hospital, Aristotle University of Thessaloniki, Thessaloniki, Greece and ${ }^{3}$ Department of Orthopedics, General Papageorgiou Hospital, Aristotle University of Thessaloniki, Thessaloniki, Greece

Correspondence: Dr A Zafeiridis, Exercise Physiology and Biochemistry Laboratory, Department of Physical Education and Sport Sciences at Serres, Aristotle University of Thessaloniki, Ag. loannis, 62110 Serres, Greece.

E-mail: zafeirid@phed-sr.auth.gr

Received 19 December 2011; revised 23 February 2012; accepted 4 March 2012; published online 10 April 2012 
of these muscles. SCI demonstrate a hypokinetic circulation during ACE, possibly attributed to venous pooling in legs due to the loss of sympathetic control, ${ }^{11}$ which may affect blood supply and consequently muscle perfusion of the upper and lower back muscles. The effect of ACE on perfusion of upper and low back muscles in SCI and able-bodied individuals remains untested. It is also unknown whether resting muscle perfusion of lower back muscles is impaired in SCI.

No previous study has assessed changes in skeletal muscle perfusion of the paralyzed legs or in the upper and lower back muscles during ACE in SCI and able-bodied individuals. This knowledge is important to elucidate into possible microvascular benefits of ACE in these muscles in SCI, as adverse changes in microcirculation in this population may be linked to health-related issues, such as increased cardiovascular risk and impaired exchange of nutrients. The aims of this study were (i) to assess muscle perfusion, at rest and during ACE, of the upper and lower back muscles and of the paralyzed leg muscles in SCI, and (ii) to compare these responses between SCI and ablebodied individuals.

\section{MATERIALS AND METHODS}

\section{Participants}

Eight SCI with complete thoracic spinal lesions $\left(\mathrm{T}_{6}-\mathrm{T}_{12}\right.$, ASIA A, time of injury $4.7 \pm 1.3$ years) and eight healthy controls (C) participated in this study. All participants had no signs of cardiovascular or metabolic disease and were not receiving medications affecting their cardiovascular system. This research has been conducted in conformity with the Declaration of Helsinki (2000) and was approved by the Institutional Ethical Committee. All participants signed the written informed consent.

\section{Experimental procedure}

On the first visit, participants performed maximal incremental test on an armergometer (Monark 881E, Varberg, Sweden). On the second visit, the participants executed a continuous $\mathrm{ACE}$ at $60 \% \mathrm{VO}_{2 \text { peak }}$ for $20 \mathrm{~min}$. All participants received injections of ${ }^{99 \mathrm{~m}} \mathrm{Tc}$-pertechnetate in the trapezius (TRAP), latissimus dorsi (LAT) and vastus lateralis (VL) muscles for measuring muscle perfusion at rest and during ACE. The clearance of ${ }^{99 \mathrm{~m}} \mathrm{Tc}$ - pertechnetate has been previously used to measure skeletal muscle perfusion at rest ${ }^{12}$ and during exercise. ${ }^{13,14}$ The local clearance of the radioactive tracer (expressed as half-life time, $\mathrm{T}_{1 / 2}$ in min) provides a good estimate of muscle perfusion and capillary flow. Comparisons of isotope clearance, allow comparisons of relative blood flow or changes in blood flow. ${ }^{13}$

The VL muscle (left side) was injected at the mid-distance between the patella and the anterior superior iliac crest with a single bolus of $0.1 \mathrm{mCi}$ of ${ }^{99 \mathrm{~m}} \mathrm{Tc}$-pertechnetate, in a total volume of $0.1 \mathrm{ml}$, using a 4 -cm long, 26-gauge needle. The radioactive counts were acquired from VL at rest for $10 \mathrm{~min}$. Next, the same amount of radioactivity was applied to the left and right TRAP and LAT muscles, using as administration points the level of the spinous process of the fourth and twelfth thoracic vertebrae for the two muscles, respectively, and the radioactive counts were recorded at rest for $10 \mathrm{~min}$. The second side was used as an internal control and the recording was performed simultaneously for the left and right TRAP and LAT muscles with the $\gamma$-camera. ACE was then performed for $10 \mathrm{~min}$, with concurrent image acquisition of TRAP and LAT. At the completion of the 10-min exercise, a second injection of a 10-fold dose (for radiopharmaceutical washout of the previous dose) of ${ }^{99 \mathrm{~m}} \mathrm{Tc}$-pertechnetate was administered to the VL using the same procedure. ACE continued with data acquisition from the VL for $10 \mathrm{~min}$. During exercise, the participants were constantly instructed to keep their trunk as stable as possible and to avoid leg movements.

A single-head $\gamma$-camera (ADAC, Genesys, Milpitas, CA, USA) equipped with a high-resolution low-energy collimator was used to record the radioactive counts because it provides the best image quality for Tc99m imaging. The camera was positioned to the closest proximity to the injection site and whole muscle (knee joint to iliac crest for the thigh region and $\mathrm{C}_{5}$ to $\mathrm{L}_{5}$ for posterior trunk) was included within the camera's field-of-view $(20 \times 15$ in $)$. Sequential images recorded the radioactive counts. The total duration of each acquisition was $10 \mathrm{~min}$, divided in 30 frames of 20 -s each. The matrix size was $128 \times 128$, in a window of $15 \%$ centered at the $140 \mathrm{KeV}$ energy of Technetium. The region of interest for the injection site was drawn, using the Philips 'Jet Stream' processing console (Workspace, Milpitas, CA, USA). The time-count curves of the 30 frames were generated from each region of interest and the isotope clearance, expressed as half-life time $\left(\mathrm{T}_{1 / 2}, \mathrm{~min}\right)$, was calculated. Absolute skeletal muscle blood flow was estimated as previously described. ${ }^{12}$

\section{Statistical analysis}

Data are presented as means \pm s.d. The physical and physiological characteristics of SCI and C were compared using independent $t$-tests. Two-way ANOVAs with repeated measures were used for examining the effects of 'group' and 'muscle' on $\mathrm{T}_{1 / 2}$ at rest, followed by Newman-Keuls post-hocs in case of significance $(\alpha=0.05)$. The same statistical approach was used for examining the effects of 'group' and 'exercise' on $\mathrm{T}_{1 / 2}$ of the tracer in each muscle group.

\section{RESULTS}

The participants' characteristics and performance data from the maximal arm-cranking test are presented in Table 1. No differences were detected between SCI and $\mathrm{C}$ in age, height, body mass and body mass index. $\mathrm{VO}_{2 \text { peak }}$ was higher in $\mathrm{C}$ than in SCI $(P<0.05)$, whereas $\mathrm{HR}_{\max }$, total exercise time and peak workload in the arm-cranking test were not different between the groups.

In SCI, the resting $\mathrm{T}_{1 / 2}$ values were shorter $(P<0.05)$ in TRAP and LAT vs VL muscle (Figure 1), whereas in $C$ there were no differences in resting $\mathrm{T}_{1 / 2}$ among the three muscles. Exercise increased the clearance rate (shorter $T_{1 / 2}$ ) of the isotope compared with rest in TRAP (Figures 2a and b, right and left side, respectively) in SCI $(P<0.001$; effect size $=3.02$ and 3.05) and in $C(P<0.001$; effect size $=3.43$ and 2.81). Similarly, there was a significant diminution of the tracer $\mathrm{T}_{1 / 2}$ during exercise compared with rest in LAT (Figures $2 \mathrm{c}$ and $\mathrm{d}$, right and left side, respectively) in SCI $(P<0.05$; effect size $=1.41$ and 1.43) and in $C(P<0.05$; effect size $=2.02$ and 2.41). Comparisons between groups did not reveal differences in $T_{1 / 2}$ at rest and during exercise in both back muscles. However, $\mathrm{T}_{1 / 2}$ in $\mathrm{VL}$ was significantly longer in SCI vs $C$ at rest $(P<0.05$; effect size $=1.66)$ and during exercise $(P<0.05$; effect size $=1.94)$. ACE had no effect on the

Table 1 Mean \pm s.d. values for physical and physiological characteristics and for the performance data of the maximal armcranking test for the individuals with a $\mathrm{SCl}$ and control participants

\begin{tabular}{|c|c|c|c|}
\hline Variable & $S C l(n=8)$ & Controls $(\mathrm{n}=8)$ & $\mathrm{P}$-value \\
\hline Age (years) & $34.3 \pm 12.2$ & $31.3 \pm 7.8$ & 0.568 \\
\hline Height $(\mathrm{cm})$ & $174 \pm 8$ & $177 \pm 11$ & 0.537 \\
\hline Body mass (kg) & $74.0 \pm 10.7$ & $82.8 \pm 16.0$ & 0.219 \\
\hline $\mathrm{BMI}\left(\mathrm{kg} \mathrm{m}^{-2}\right)$ & $24.6 \pm 3.9$ & $26.2 \pm 3.1$ & 0.352 \\
\hline $\mathrm{HR}_{\text {rest }}\left(\mathrm{b} \min ^{-1}\right)$ & $67.9 \pm 6.9$ & $65.5 \pm 4.7$ & 0.433 \\
\hline $\mathrm{SBP}_{\text {rest }}(\mathrm{mm} \mathrm{Hg})$ & $120.0 \pm 15.9$ & $127.4 \pm 12.3$ & 0.318 \\
\hline $\mathrm{DPB}_{\text {rest }}(\mathrm{mm} \mathrm{Hg})$ & $71.3 \pm 10.8$ & $71.3 \pm 6.4$ & 1.000 \\
\hline $\mathrm{MAP}_{\text {rest }}(\mathrm{mm} \mathrm{Hg})$ & $87.5 \pm 11.7$ & $90.0 \pm 7.8$ & 0.628 \\
\hline $\mathrm{VO}_{2 \text { peak }}\left(\mathrm{ml} \mathrm{kg}^{-1} \mathrm{~min}^{-1}\right)$ & $18.2 \pm 2.6$ & $22.8 \pm 5.3^{*}$ & 0.047 \\
\hline $\mathrm{HR}_{\max }\left(\mathrm{b} \min ^{-1}\right)$ & $170 \pm 11$ & $174 \pm 14$ & 0.530 \\
\hline Total exercise time (min) & $8.3 \pm 1.2$ & $9.9 \pm 1.9$ & 0.060 \\
\hline Peak workload (watts) & $87 \pm 17$ & $103 \pm 23$ & 0.129 \\
\hline
\end{tabular}

Abbreviations: $\mathrm{BMI}$, body mass index; DBP, diastolic blood pressure; HR, heart rate; MAP, mean arterial pressure; $\mathrm{SBP}$, systolic blood pressure; $\mathrm{SCl}$, spinal cord injury; $\mathrm{VO}_{2}$, oxygen consumption.

${ }^{*} \mathrm{P}<0.05 \mathrm{SCl}$ vs $\mathrm{C}$. 


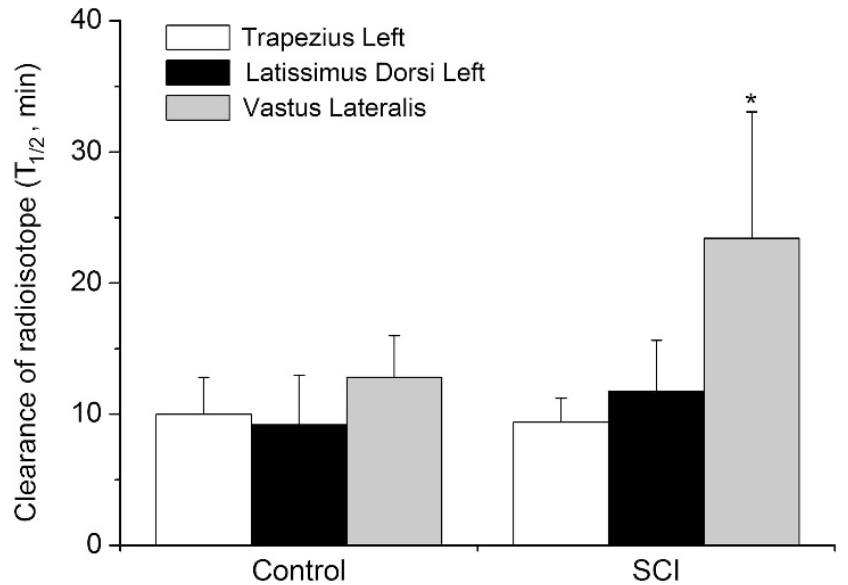

Figure 1 The mean ( \pm s.d.) values for resting muscle perfusion (clearance rate of radioactive tracer presented as half-life time, $\mathrm{T}_{1 / 2}$, in $\mathrm{min}$ ) in trapezius, latissimus dorsi and vastus lateralis muscles in $\mathrm{SCl}$ and control individuals. ${ }^{*} P<0.05$ vs trapezius and latissimus dorsi muscles within $\mathrm{SCl}$.

radioisotope clearance $\left(\mathrm{T}_{1 / 2}\right)$ in VL in both groups (Figure 2e). Table 2 depicts the skeletal muscle blood flow data in TRAP, LAT and VL muscles at rest and during exercise in the two groups.

\section{DISCUSSION}

The novel aspects of this study are that it examined the microcirculation of upper and lower back and leg muscles during rest in SCI and able-bodied individuals, and explored whether ACE can promote blood perfusion in these muscles to induce favorable microvascular and muscular adaptations. The major findings are: (i) resting muscle perfusion of VL is lower in SCI compared with able-bodied individuals, whereas it remains intact in upper and lower back muscles in SCI, (ii) sub-maximal ACE increases blood perfusion of upper and lower back muscles in SCI and able-bodied individuals, but it does not promote significant increases in muscle perfusion of the lower limbs and (iii) the magnitude of muscle perfusion response in upper and lower back muscles during sub-maximal ACE is similar in SCI and able-bodied individuals, suggesting that blood flow distribution to these muscles is intact during ACE in paraplegics with thorasic injury.

\section{Resting muscle perfusion}

Resting muscle perfusion of VL was reduced in SCI compared with able-bodied individuals by $\sim 40 \%$. This magnitude of reduction in perfusion of VL in SCI is in accordance with the reduction (35-70\%) previously reported in studies that measured whole limb flow, conduit artery flow, or peak reactive hyperemic response., ${ }^{2,7,8}$ Several physiological observations may collectively explain the reduced resting muscle perfusion of paralyzed limbs in SCI. First, skeletal muscle perfusion is mainly regulated by microcirculation. Second, physical inactivity and SCI induce structural changes in resistance vessels resulting in increased vascular resistance in SCI. ${ }^{2}$ Third, SCI present a reduced capillary diameter and fewer capillaries, ${ }^{1,15}$ partially as a result of muscle atrophy and disuse. Finally, the abnormal muscle geometry in SCI may also cause obstruction in microvasculature and reduce muscle perfusion. ${ }^{1}$

The finding of reduced resting microcirculation in VL of SCI is an important health-related issue, as chronically reduced blood flow may impair vessel structure and function. Changes in microvasculature may be linked to adverse alterations in muscle fiber morphology, morphometry, ultrastructure and composition in SCI. ${ }^{15}$ Chronic muscle hypoperfusion may result in increased systemic vascular resistance and in reduced glucose uptake by the muscle, and thus, in increased risk for developing cardiovascular disease and type 2 diabetes. Impaired microcirculation may also reduce amino-acid delivery to muscle tissue contributing to muscle mass loss due to physical inactivity.

\section{Muscle perfusion during arm exercise}

This is the first attempt to study lower-limb muscle perfusion during ACE in SCI and in able-bodied individuals. ACE did not effectively increase blood perfusion of VL in either group. Previous studies that examined blood flow to the legs of SCI or able-bodied individuals during ACE assessed changes in limb volume or in femoral artery and cutaneous circulation. One study reported a minimal increase in SCI and able-bodied individuals in femoral artery blood flow, ${ }^{7}$ whereas, others reported a small decrease in calf volume ${ }^{5}$ or no changes in cutaneous and femoral artery blood flow in SCI. ${ }^{6,8}$ In agreement with those reports showing unchanged blood flow in lower limbs of SCI and able-bodied individuals during ACE, we document no effect of $\mathrm{ACE}$ at the level of muscle microcirculation. It appears that moderateintensity aerobic exercise involving relatively small muscle mass does not induce a systemic hyperemic response.

The systemic effects of exercise to non-exercising muscles may be promoted when exercise elicits significant changes in cardiac output, arterial pressure, and/or circulating humoral mediators. ${ }^{16}$ It is possible that the protocol used in this study, in terms of intensity or muscle mass, did not reach a threshold for hemodynamic forces and/or circulating factors that would result in a hyperemic response in leg muscles. Although a more intense ACE protocol could have increased blood perfusion of VL in SCI, the adherence to such an intensive exercise program would have been uncertain.

Recent findings in humans suggested that hyperemia is essential for inducing microvascular adaptations. ${ }^{3}$ Thus, the results of this study propose that a single bout of $\mathrm{ACE}$ at $60 \% \mathrm{VO}_{2 \text { peak }}$ for $20 \mathrm{~min}$ may not promote microvascular adaptations in leg muscles of SCI and ablebodied individuals. However, in a recent cross-sectional study, although only localized effects of training were reported for caliber size of conduit and resistance arteries, the impact of training on arterial wall thickness was systemic and was observed beyond the exercised muscles. ${ }^{17}$ There is also evidence that oxygen delivery and cutaneous microcirculation in legs may be improved following ACE. ${ }^{18,19}$ Therefore, the possibility that favorable microvascular adaptations might occur in leg muscles of SCI after a long-term ACE, should be further evaluated. Indeed, animal studies have shown that exercise training induces vascular adaptations in skeletal muscles even without elevations in blood flow or metabolism. ${ }^{16}$ Additionally, following one-limb training, capillary growth ${ }^{4}$ and increased resting blood flow ${ }^{20}$ was observed in the passively moved ${ }^{4}$ and in the untrained limb. ${ }^{4,20}$

This is the first study that monitored blood perfusion of back muscles in SCI during ACE. Muscle perfusion increased in TRAP and LAT during ACE in SCI and able-bodied individuals suggesting that ACE may promote adaptations within the microvascular bed of upper and lower back muscles. ${ }^{3}$ This is of particular relevance for SCI, as lower back muscles are important for maintaining posture and stability of the spine, as well as during the wheelchair propulsion and sitting pivot transfers. ${ }^{9}$ We observed a similar exercise-induced response in muscle perfusion of back muscles in the two groups, demonstrating that the microvascular hemodynamics are intact in these muscles in SCI with thoracic injury and that blood flow delivery 

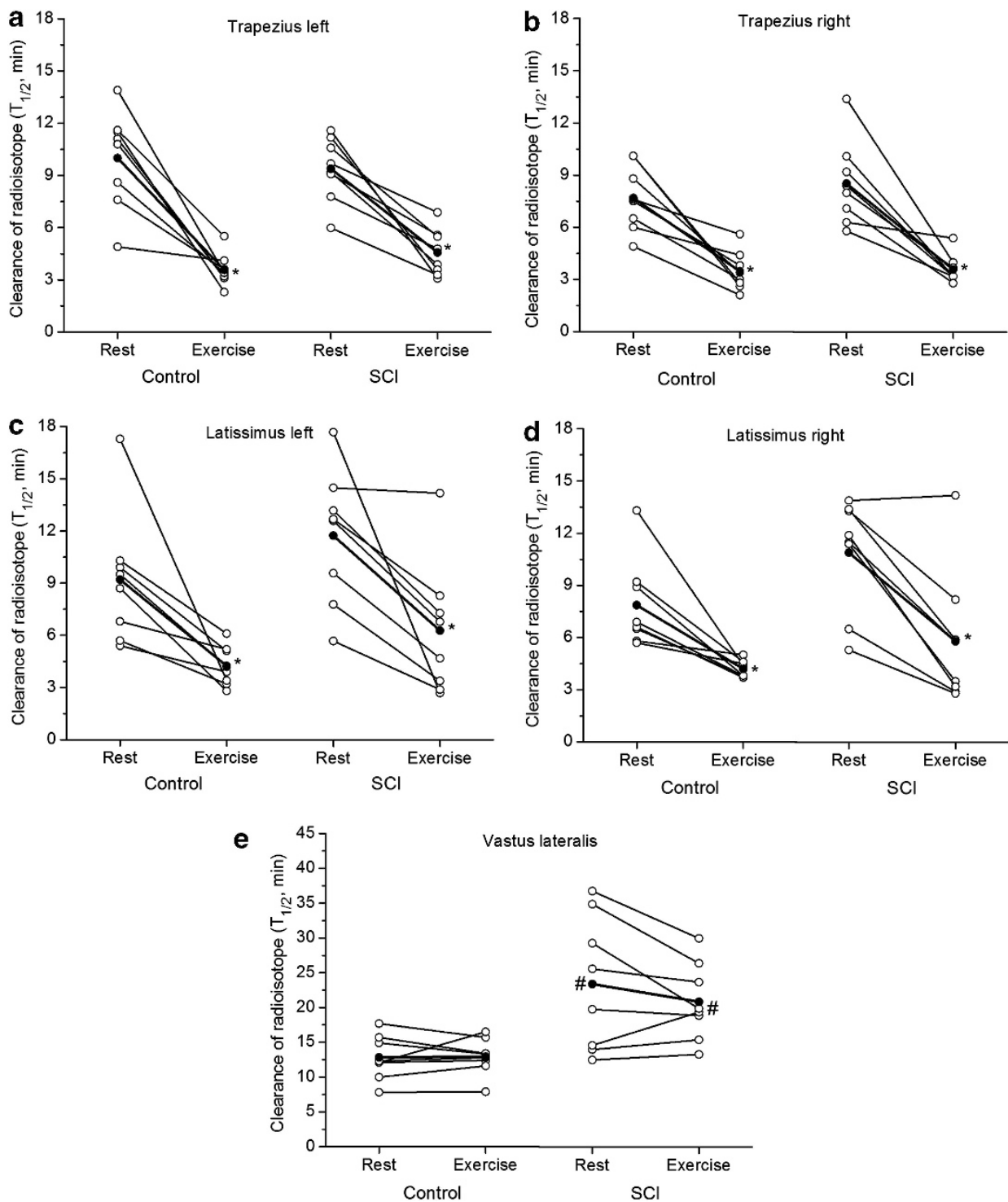

Figure 2 The individual (open circles) and mean values (filled circles) for muscle perfusion index (clearance of radioactive tracer presented as half-life time, $\mathrm{T}_{1 / 2}$, in $\mathrm{min}$ ) during rest and exercise in $\mathrm{SCl}$ and control individuals in trapezius ((a) and (b) for left and right sides, respectively), latissimus dorsi ((c) and (d) for left and right sides, respectively), and vastus lateralis muscles (e). ${ }^{*} P<0.05$ vs rest within the group; \#P<0.05 between $\mathrm{SCl}$ and control within rest and exercise.

Table 2 Mean \pm s.d. values for skeletal muscle blood flow $\left(\mathrm{ml} \cdot \mathrm{min}^{-1}\right.$ per $100 \mathrm{~g}$ ) in trapezius, latissimus dorsi and vastus lateralis muscles at rest and during sub-maximal exercise in individuals with a $\mathrm{SCl}$ and control participants.

\begin{tabular}{|c|c|c|c|c|}
\hline & \multicolumn{2}{|c|}{ Left side } & \multicolumn{2}{|c|}{ Right side } \\
\hline & $S C l(n=8)$ & Control $(n=8)$ & $S C l(n=8)$ & Control $(n=8)$ \\
\hline Rest & $5.30 \pm 1.26$ & $5.26 \pm 2.03$ & $5.97 \pm 1.53$ & $6.58 \pm 1.73$ \\
\hline Exercise & $11.20 \pm 3.10^{*}$ & $14.12 \pm 3.45^{*}$ & $13.83 \pm 2.52^{*}$ & $15.03 \pm 4.51^{*}$ \\
\hline \multicolumn{5}{|c|}{ Latissimus dorsi } \\
\hline \multicolumn{5}{|c|}{ Vastus lateralis } \\
\hline Rest & $2.39 \pm 1.00$ & $3.96 \pm 1.09^{* *}$ & & \\
\hline Exercise & $2.44 \pm 0.66$ & $3.86 \pm 0.97^{* *}$ & & \\
\hline
\end{tabular}

Abbreviation: $\mathrm{SCl}$, spinal cord injury.

${ }^{*} P<0.05$ vs respective rest; ${ }^{* *} P<0.05$ vs respective SCI. 
to upper and lower back muscles is preserved in SCI during submaximal ACE. This is not an entirely expected finding, as the lack of sympathetic control in SCI may increase venous pooling in lower limbs during ACE, limiting stroke volume and ultimately blood delivery to the active tissue. Although the increase in heart rate may compensate for the reduced stroke volume and maintain cardiac output in SCI during ACE, it has been demonsrated that cardiac output remains consistently lower in SCI despite the increased heart rate. ${ }^{11}$ Our findings of intact upper and lower back muscles circulation in SCI provide further support that inactivity is the main source of microvascular maladaptations and muscle hypoperfusion in the paralyzed legs of SCI.

In conclusion, leg muscle perfusion is reduced in SCI, whereas there is no evidence of impaired microcirculation in upper and lower back muscles. ACE increased hyperemia in upper and lower posterior trunk muscles of SCI and able-bodied individuals to a similar extent, implying no circulatory insufficiency within these muscles during exercise in paraplegics. Thus, continuous ACE at $60 \% \mathrm{VO}_{2 \text { peak }}$ appears as an effective exercise mode to promote microvascular adaptations and improve blood supply and performance of upper and lower posterior trunk muscles in SCI. However, continuous ACE at 60\% was not sufficient to induce a hyperemic response in VL in paraplegics. These results point towards an alternative training strategy (that is, intermittent arm exercise of higher intensity and/or resistance exercise) that may elicit greater systemic hemodynamic and/or humoral responses to reverse/delay the microvascular maladaptations within the paralyzed legs of SCI. Yet, we cannot exclude with certainty that a long-term armcranking training will not result in beneficial structural and/or functional microvascular adaptations in paralyzed muscles of SCI.

\section{DATA ARCHIVING}

There were no data to deposit.

\section{CONFLICT OF INTEREST}

The authors declare no conflict of interest.

\section{ACKNOWLEDGEMENTS}

The authors would like to thank all the participants for their cooperation and their voluntary participation in this study.
1 Ruschkewitz Y, Gefen A. Cellular-scale transport in deformed skeletal muscle following spinal cord injury. Comput Methods Biomech Biomed Engin 2011; 14 411-424.

2 Hopman MT, Groothuis JT, Flendrie M, Gerrits KH, Houtman S. Increased vascular resistance in paralyzed legs after spinal cord injury is reversible by training. J Appl Physiol 2002; 93: 1966-1972.

3 Green DJ, Spence A, Halliwill JR, Cable NT, Thijssen DH. Exercise and vascular adaptation in asymptomatic humans. Exp Physiol 2011; 96: 57-70.

4 Hoier B, Rufener N, Bojsen-Moller J, Bangsbo J, Hellsten Y. The effect of passive movement training on angiogenic factors and capillary growth in human skeletal muscle. J Physiol 2010; 588: 3833-3845.

5 Hopman MT, Verheijen PH, Binkhorst RA. Volume changes in the legs of paraplegic subjects during arm exercise. J Appl Physiol 1993; 75: 2079-2083.

6 Muraki S, Yamasaki M, Ehara Y, Kikuchi K, Seki K. Effect of maximal arm exercise on skin blood flux in the paralyzed lower limbs in persons with spinal cord injury. Eur $J$ Appl Physiol Occup Physiol 1996; 74: 481-483.

7 Thijssen DH, Green DJ, Steendijk S, Hopman MT. Sympathetic vasomotor control does not explain the change in femoral artery shear rate pattern during arm-crank exercise. Am J Physiol Heart Circ Physiol 2009; 296: H180-H185.

8 Thijssen DH, Steendijk S, Hopman MT. Blood redistribution during exercise in subjects with spinal cord injury and controls. Med Sci Sports Exerc 2009; 41: 1249-1254.

9 Yang YS, Koontz AM, Triolo RJ, Mercer JL, Boninger ML. Surface electromyography activity of trunk muscles during wheelchair propulsion. Clin Biomech 2006; 21 1032-1041.

10 Bjerkefors A, Carpenter MG, Cresswell AG, Thorstensson A. Trunk muscle activation in a person with clinically complete thoracic spinal cord injury. Rehabil Med 2009; 41 390-392.

11 Jacobs PL, Mahoney ET, Robbins A, Nash M. Hypokinetic circulation in persons with paraplegia. Med Sci Sports Exerc 2002; 34: 1401-1407.

12 Brown SL, Hunt JW, Hill RP. A comparison of the rate of clearance of xenon (133Xe) and pertechnetate ion (99mTc04-) in murine tumors and normal leg muscles. Int J Rad Appl Instrum B 1988; 15: 381-390.

13 Cutajar CL, Brown NJ, Marston A. Muscle blood-flow studies by the technetium $(99 \mathrm{mTc})$ clearance technique in normal subjects and in patients with intermittent claudication. Br J Surg 1971; 58: 532-537.

14 Seto M, Bunko H, Shuke N, Takahashi K, Sakaibori Y, Terada H et al. Quantitative regional blood flow measurements in exercising leg skeletal muscle based on $99 \mathrm{mTc}$ pertechnetate clearance. Nucl Med Commun 2008; 29: 770-774.

15 Scelsi R. Skeletal muscle pathology after spinal cord injury: our 20 year experience and results on skeletal muscle changes in paraplegics, related to functional rehabilitation. Basic Appl Myol 2001; 11: 75-85.

16 Padilla J, Simmons GH, Bender SB, Arce-Esquivel AA, Whyte JJ, Laughlin $\mathrm{MH}$. Vascular effects of exercise: endothelial adaptations beyond active muscle beds. Physiology 2011; 26: 132-145.

17 Rowley NJ, Dawson EA, Birk GK, Cable NT, George K, Whyte G et al. Exercise and arterial adaptation in humans: uncoupling localized and systemic effects. J Appl Physiol 2011; 110: 1190-1195.

18 Tew G, Nawaz S, Zwierska I, Saxton JM. Limb-specific and cross-transfer effects of arm-crank exercise training in patients with symptomatic peripheral arterial disease. Clin Sci 2009; 117: 405-413.

19 Klonizakis M, Winter E. Effects of arm-cranking exercise in cutaneous microcirculation in older, sedentary people. Microvasc Res 2011; 81: 331-336.

20 Shen M, Gao J, Li J, Su J. Effect of ischaemic exercise training of a normal limb on angiogenesis of a pathological ischaemic limb in rabbits. Clin Sci 2009; 117 201-208. 